\title{
ADVANCED AUTOMATION IN TEXTILE INDUSTRY PRODUCTION LINES
}

\author{
Michail Papoutsidakis \\ Dept. of Industrial Design and \\ Production Engineering, \\ University of West Attica \\ Athens, Greece
}

\author{
Dimitrios Piromalis \\ Dept. of Industrial Design and \\ Production Engineering, \\ University of West Attica \\ Athens, Greece
}

\author{
Georgios Priniotakis \\ Dept. of Industrial Design and \\ Production Engineering, \\ University of West Attica \\ Athens, Greece
}

\begin{abstract}
Textile industry is one of the few basic industries that have always been a necessary component of human life. In any study of textile fabrics the meaning of the word textile must be made clear. The dictionary states that the word is derived from the Latin word 'texere' to weave, but a wider meaning than simply that of weaving must be accepted since that is only one of various ways of making textile fabrics. One may classify it as a more glamorous industry, but whatever it is, it provides with the basic requirement called clothes. The researcher has given in this chapter all possible terms and concepts involved in textile production process. Most of these terms are technical. This chapter is an attempt to understand clear cut ideas about research topic. It gives an insight about terms that are used all around the world in context of textile industry. There are numerous kinds of fibres and other raw materials, which are used to produce a cloth. But most of them are unheard of. This chapter is designed in such a way that it is easy to understand the basics of textiles.
\end{abstract}

Keywords-Textile industry, spinning, weaving, garmenting, dyeing, automation using, effects of automation, future textile machinery, employment

\section{INTRODUCTION}

This paper reviews the automation which has taken place in the textile industry in the recent past which has helped the industry in improving the productivity and quality of the textile products. The paper mentions various textile processes and the automation which has taken in these processes. The paper also highlights various benefits which have been achieved through automation in the textile industry. . One may classify it as a more glamorous industry, but whatever it is, it provides with the basic requirement called clothes. This chapter is about textiles. The researcher has given in this chapter all possible terms and concepts involved in textile production process. Most of these terms are technical. This chapter is an attempt to understand clear cut ideas about research topic. It gives an insight about terms that are used all around the world in context of textile industry. There are numerous kinds of fibres and other raw materials, which are used to produce a cloth. But most of them are unheard of. This chapter is designed in such a way that it is easy to understand the basics of textiles.

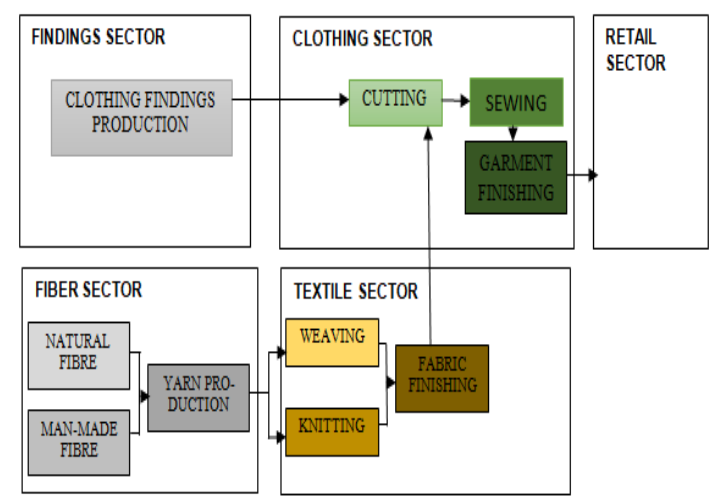

Fig. 1. Automation Textile Industry

\section{SPINNING}

Automation in spinning has taken place in various process like picking and ginning which were completely manual earlier. High Volume Instrument (HVI) system has made possible to carry out the cotton fiber test in seconds which used to take hours before. HVI tests have improved the accuracy in measuring the cotton's staple lengths, colour grade, micronaire, strength, elongation and uniformity index. Cotton mixing has been automated so that the uniformity can be achieved in the Yarn. Blow-room performance has been improved these days by using sequence of different machines required or opening, cleaning and blending which are arranged in series and are connected by transport ducts. Automation is recently done to separate out the contamination of any colour, size and nature in the fibre. Machines using ultraviolet, optic and acoustic technologies are being used for the detection and elimination of contaminant of any colour, size and nature thus improving the overall quality of the final yarn produced. 


\section{International Journal of Engineering Applied Sciences and Technology, 2019 \\ Vol. 4, Issue 5, ISSN No. 2455-2143, Pages 504-507 \\ Published Online September 2019 in IJEAST (http://www.ijeast.com)}

Automation has been achieved in spinning by the invention of machines like ring spinning, air-jet spinning, rotor spinning, Vortex spinning etc. Improvements in Ring spinning machines have taken place through drive systems, drafting systems and use of robotics. Yarn fault detection has been automated now to improve the production and to get the uniform yarn quality. Yarn knots have now been replaced with the joints using splicing techniques like air splicing, wet splicing, hot air splicing and moist air splicing which minimizes the defects in final fabric thus produced. Transport automation is done using robots to carry out heavy takes which were earlier done by humans. Package collection from the spinning mills and even palletizing and packaging is being done these days using automated solutions. All these automation in the spinning process has reduced the need of the skilled manpower.

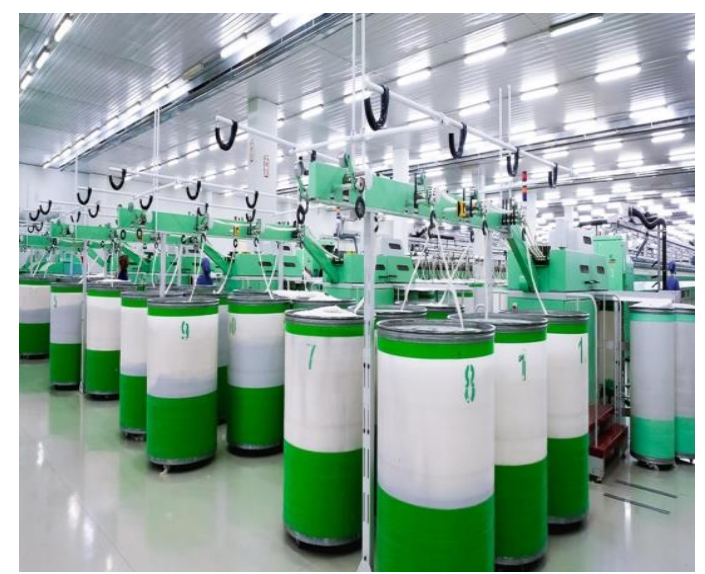

Fig. 2. Spinning Machine

\section{WEAVING}

Weaving machines have witnessed lot of improvement in last three decades resulting in improved quality and production. Major development like automatic shuttle and shuttleless looms has taken the industry to a different level. Shuttleless machines have made possible the production of fault free cloth efficiently. Developments have been done for shuttleless machines in three basic picking principles, i.e. rapier, projectile, air-jet and water-jet The key features of shuttleless machines are higher speed, wider width, automatic pick finding, microprocessor controls, quick style change etc. Various improvements have taken place recently in rapier looms like development in filling insertion, shedding mechanism, let-off mechanism, take-up mechanism, selvedge, quick style change etc. Microprocessors are now integrated with the weaving machines which monitor, control, regulate and optimize all key aspects of the weaving machines.

Automation has been achieved with the help of microelectronics to control warp tension, picking of multi- filling colours, break detection data collection etc. which has helped in improving the production as a lesser labour cost.

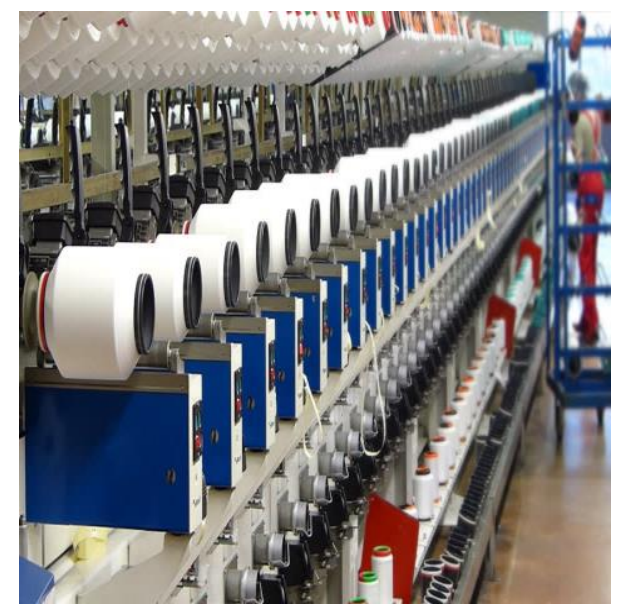

Fig. 3. Weaving Factory

\section{GARMENTING}

Garmenting has undergone many advancements in the recent past. Technologies being employed in automatic garment manufacturing are reconfigurable robotic handling devices, cutting table with automatic unloading, intelligent transportation system, manufacturability prediction, Virtual TryOn, 3D garment design etc. Robots handling devices are being used in the garment industry for automated handling of the cut fabric parts where they are collected and delivered to the automated transport system for transferring it to the next stage.

\section{DYEING}

Dyeing process has multiple stages e.g. Desizing, Scouring, Bleaching, Printing and Finishing. Automation helps the dyeing process by providing the precise control on various factors which are critical for the quality like pressure, temperature, time of treatment, water level etc. Robots are being used to pick the yarn bobbins and transport them with the help of the self-propelled bobbin carriers to the dyeing and drying machines. Unloading is also automated with the help of robots. Automation in dyeing helps in reduced water consumption and lesser cost for treatment and water. It also helps in controlling the consumption of colour chemicals and hence increased utilisation of man and machine and production per shift. It also automates the colour matching in the dyeing process which increases the uniformity of the end products 


\section{International Journal of Engineering Applied Sciences and Technology, 2019 Vol. 4, Issue 5, ISSN No. 2455-2143, Pages 504-507 \\ Published Online September 2019 in IJEAST (http://www.ijeast.com)}

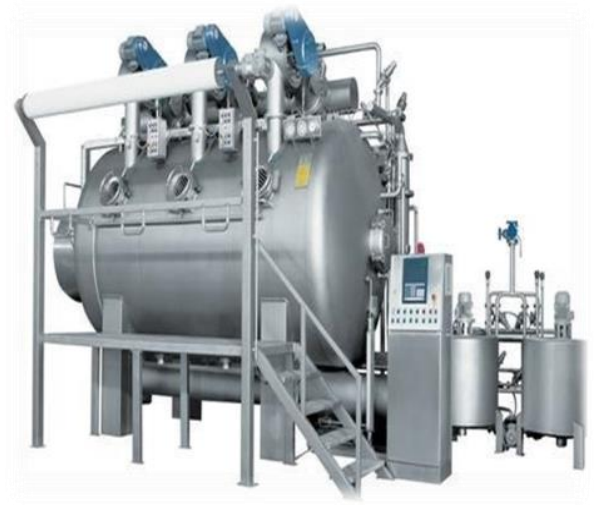

Fig. 4. HT Synchron Airjet Fabric Dyeing Machine

\section{AUTOMATION USING IT}

ERP systems are widely being used in the textile industry for managing the Inventory, Orders, Production, Logistics, Sales and Marketing and Financials etc. Many generic ERP solutions as well as customised ERP solutions are available for textile industry. ERP solution helps in integrating the operational processes and establish synergies between various resources of the textile industries. Automated Systems are also being developed for the effective communication between the production floor and other supervisory control systems. Now a days, operating and process related data is being captured from the machines and is being analysed in various BI software for the operations analysis and further productivity improvement. $\mathrm{BI}$ reports are being published for the top management and are used for various decision making processes.

\section{EFFECTS OF AUTOMATION}

Automation brings in quality and also improves the productivity in the mills and garment industry. Productivity increase because of automation has resulted in reduction in the overall manpower in the textile industry. Figure 2 shows the overall decline in the manpower in Mills, Mills Products and Apparel industry in last 15 Years because of the productivity enhancements brought by the automation.

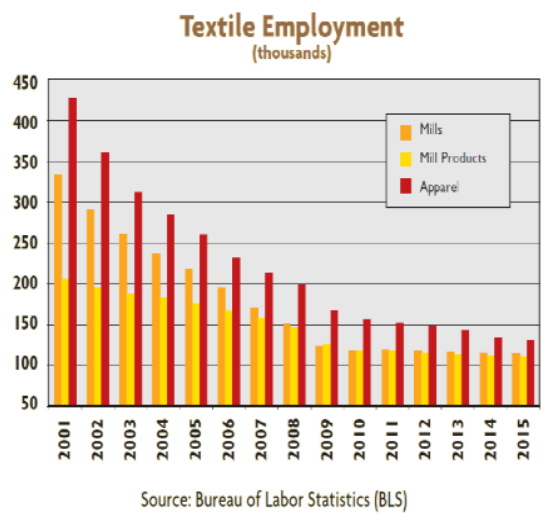

Fig. 5. Textile Employment in US

\section{FUTURE TEXTILE MACHINERY}

Research and development is being done in the textile machinery to achieve further automation and enhancements. Emphasis is to further improve the quality and production and at the same time bring down the costs. Advancements are taking place to reduce the space and power requirements for various textile machinery, increase their speed and efficiency. Big data and Internet of Things is also going to play a big role in future textile machinery to analyse the machine behaviour and proactively take decisions to improve the quality and productivity of the machines.

\section{CONCLUSION}

Textile Finishing covers an extremely wide range of activities which are performed on textiles before they reach the final customer. The term finishing includes all the mechanical and chemical processes employed commercially to improve the acceptability of the product.

Mechanical finishes:

1.Calendering:

Compression of fabric between two heavy rollers to give a smooth appearance to the surface of the fabric.

\section{Napping and Shearing:}

They are applied to raise a velocity or soft surface. Shearing cuts the raised naps to a uniform height.

Chemical finishes: (involve application of chemicals)

1.Water Repellency.

2.Flame retardancy.

3.Resin Finishing Anticrease, Wash-n-wear, durable press (permanent pleating).

4.Softening (handle modification). 


\section{International Journal of Engineering Applied Sciences and Technology, 2019 \\ Vol. 4, Issue 5, ISSN No. 2455-2143, Pages 504-507 \\ Published Online September 2019 in IJEAST (http://www.ijeast.com)}

5.Oil and soil repellancy.

6.Antistatic finishes.

7.Anti-microbial finish.

8.Moth proofing and insect damage.

Automation technologies has helped the textile industry to increase the output multiple times that too at a cheaper cost. Automation products and solutions are available now not only for the individual process or machine, but for the entire production line. Some of the key benefits achieved through automation are:

- Improved production at cheaper cost

- Better quality

- Safety for the humans and machines

- Predictable production and inventory

- Energy savings

- Lower impact on environment

- Better machine uptimes

- Self-diagnostics and Predictive maintenance

- Efficient packaging and transport

- Improved customer satisfaction.

\section{$\mathrm{X}$. ACKNOWLEDGMENTS}

All authors would like to thank the University of West Attica for the financial support provided to them for this research project.

\section{REFERENCES}

[1] Tait N. 1996. Materials handling in the garment factory. Apparel International Position and Suction Control of a Reconfigurable Robotic Gripper. Machine Intelligence and Robotic Control.

[2] Zöll K. 2002.Sewing technology for manufacturing of textile covers. Dissertation Shaker Verlag, Aachen

[3] Zöll K. 2003a. Potentials provided by robot supported sewing. J DNZ fashion industry international, Bielefeld.

[4] Zöll K. 2003b. Three dimensional sewing. J Technical Textiles, Frankfurt

[5] Moll P, Händler K. 1997. New ideas and concepts for the sewing industry. In: Annual Book for garment industry, Schiele \& Schön, Berlin.

[6] Moll P, Schütte U. 1997. New standards for the generation of three dimensional covers. On: 26th Textile Research Symposium Mt. Fuji Japan. 